Cahiers de la recherche sur les droits

Cahiers

Fur les Droits fondamentaux

6 | 2008

Pouvoirs exceptionnels et droits fondamentaux

\title{
Régimes légaux en période exceptionnelle et régimes exceptionnels en période normale
}

Gilles Armand

\section{OpenEdition}

Édition électronique

URL : https://journals.openedition.org/crdf/6867

DOI : $10.4000 /$ crdf.6867

ISSN : 2264-1246

Éditeur

Presses universitaires de Caen

Édition imprimée

Date de publication : 31 décembre 2008

Pagination : 113-122

ISBN : 978-2-84133-259-5

ISSN : $1634-8842$

Référence électronique

Gilles Armand, «Régimes légaux en période exceptionnelle et régimes exceptionnels en période normale », Cahiers de la recherche sur les droits fondamentaux [En ligne], 6 | 2008, mis en ligne le 11 décembre 2020, consulté le 17 novembre 2022. URL : http://journals.openedition.org/crdf/6867 ; DOI https://doi.org/10.4000/crdf.6867 


\title{
Régimes légaux en période exceptionnelle et régimes exceptionnels en période normale
}

\author{
Gilles ARMAND \\ Maître de conférences en droit public à I'Université de Caen Basse-Normandie, CRDFED
}

\author{
I. L'extension des pouvoirs exceptionnels \\ A. Des pouvoirs extensibles \\ 1. La politique de l'amalgame \\ 2. La contamination du droit commun par le droit dérogatoire \\ B. Des pouvoirs étendus \\ 1. Des pouvoirs à dimension exponentielle \\ 2. Des pouvoirs à géométrie variable
}

II. L'affaiblissement de la garantie judiciaire

A. Une réduction de la garantie judiciaire

1. Des pouvoirs exceptionnels sans contrôle judiciaire

2. Des pouvoirs exceptionnels sous contrôle administratif

B. Un appauvrissement de la garantie judiciaire

1. L'appauvrissement de l'autorité judiciaire

2. L'appauvrissement du contrôle judiciaire

Dans l'un des passages les plus célèbres de son œuvre De l'esprit des lois, Montesquieu a pu affirmer « qu'il y a des cas où il faut mettre, pour un moment, un voile sur la liberté, comme l'on cache les statues des dieux » ${ }^{1}$. Cette affirmation correspond parfaitement à l'état d'exception au sens classique, "entendu comme un moment pendant lequel les règles de droit prévues pour des périodes de calme sont transgressées, suspendues ou écartées pour faire face à un péril $»^{2}$. Les droits et les libertés sont les principales victimes de cette "jurislation" exceptionnelle qui entraîne leur suspension temporaire, conformément aux prescriptions de l'article 15 de la Convention européenne de sauvegarde des droits de l'homme et des libertés fondamentales $(\mathrm{CEDH})^{3}$, auxquelles font écho les régimes français de l'état de siège et de l'état d'urgence ${ }^{4}$.

En reprenant Montesquieu, on doit cependant garder bien présent à l'esprit que ce n'est que « pour un moment » que le voile doit être mis sur la liberté. Comme l'a remarquablement souligné le Conseil d'État dans son ordonnance Allouache du 9 décembre $2005^{5}$, « dans un État de

1. Montesquieu, De l'esprit des lois, Livre XII, chapitre 19.

2. Voir, dans le présent numéro, l’article de F. Saint-Bonnet, «L'état d'exception et la qualification juridique ».

3. Sur ce point, voir, dans le présent numéro, l'article de G. Gonzalez, «L'état d’urgence au sens de l'article 15 de la Convention européenne des droits de l'homme».

4. Voir, dans le présent numéro, l'article de G. Lebreton, «Les atteintes aux droits fondamentaux par l'état de siège et l'état d'urgence ».

5. CE, Ord. Réf., 9 décembre 2005, $\mathrm{M}^{\mathrm{me}}$ Allouache et autres, Rec., p. 499. 
droit » il est en effet de la «nature » même d'un « régime de pouvoirs exceptionnels » d'être limité, non seulement "dans l'espace», mais aussi et surtout «dans le temps». Et c'est certainement dans la volonté du juge administratif d'éviter que le voile ne se transforme en chape de plomb définitive sur les droits fondamentaux, qu'il faut rechercher la justification de l'extension de son contrôle à la nécessité de la mise en place et du maintien de l'état d'urgence.

Mais parallèlement, ou en marge, des régimes légaux ${ }^{6}$ en période exceptionnelle, on a assisté, en France comme ailleurs ${ }^{7}$, au développement de régimes exceptionnels en période normale. Face à une menace, réelle ou supposée, venue principalement de l'extérieur et dont le terrorisme constitue le symbole le plus retentissant, ces régimes se traduisent par la mise en place de mécanismes particuliers de prévention et de répression des atteintes à l'ordre public conférant aux autorités de police des pouvoirs exorbitants du droit commun. Or, bien qu'ils soient souvent adoptés en urgence et dans un contexte spécifique, ces régimes contemporains ont une nature potentiellement permanente qui les distingue de l'état d'exception traditionnel, par essence provisoire.

La loi du 15 novembre 2001 relative à la sécurité quotidienne ${ }^{8}$ est topique de cette évolution, elle qui, frappée de plein fouet par les attentats du 11 septembre, devint une loi antiterroriste, sorte de Patriot Act, version soft à la française, et dont les dispositions censées être programmées pour une durée de vie limitée au 31 décembre 2003, ont finalement été prolongées ad vitam aeternam par les lois Sarkozy I du 18 mars $2003^{9}$ et Perben II du 9 mars $2004^{10}$. Et comment ne pas croire que l'énième (dernière?) loi relative à la lutte contre le terrorisme du 23 janvier $2006^{11}$, durcie par son adoption en pleine crise des banlieues, subisse le même sort au moment du rendez-vous fixé avec le Parlement au 31 décembre $2008^{12}$.

Puisqu'on assiste à une "permanence du temporaire ${ }^{13}$ et que les nouveaux régimes exceptionnels sont apparemment affranchis de toute contrainte temporelle, ils doivent être limités dans leur étendue et leur portée et voir leur mise en ouvre assortie de garanties juridiques, faute de quoi on pourrait assister, sous l'effet d'un mouvement de généralisation incontrôlé, à l'instauration d'un «état d'exception permanent ${ }^{14}$, conçu comme « un paradigme normal de gouvernement ${ }^{15}$ et rendant inutiles, car dépassés, les pouvoirs prévus par les régimes légaux applicables en période exceptionnelle. En France, la liberté individuelle apparaît apte à constituer ce seuil infranchissable, en période normale, par le législateur: parce que matériellement, il existe des " principes essentiels » ${ }^{16}$ qui s'opposent à ce que cette liberté fasse l'objet d'atteintes générales et absolues, ou encore imprécises et discrétionnaires, de la part du législateur et que, d'un point de vue procédural ou formel, elle est placée en vertu de l'article 66 de la Constitution sous la garde de l'autorité judiciaire ${ }^{17}$. Pour prendre l'exemple le plus significatif, ces garanties complémentaires viendraient s'opposer à ce que le législateur habilite les autorités administratives à pratiquer des perquisitions de jour comme de nuit nonobstant toute intervention préalable de l'autorité judiciaire, comme le prévoit l'article 11 de la loi du 3 avril 1955 sur l'état d'urgence, dans la mesure où il s'agirait d'une dénaturation de la liberté individuelle interdite, hors période exceptionnelle, par la Constitution.

Mais pour que la réalité colle à la théorie, encore faudrait-il que la contrainte de constitutionnalité trouve un juge pour la faire respecter. Or on peut se demander si sous les assauts répétés des législateurs, de droite comme de gauche $^{18}$, soutenus par une opinion publique avide de sécurité $^{19}$, le Conseil constitutionnel n'a pas renoncé à exercer cette fonction. Depuis la décision du 13 mars 2003 relative à la loi pour la sécurité intérieure ${ }^{20}$ jusqu'à celle du 19 janvier 2006 portant sur la loi relative à la lutte contre le terrorisme ${ }^{21}$, le contentieux français est en effet marqué par un affaiblissement constant de la contrainte de constitutionnalité révélé par la mise en œuvre d'un contrôle restreint en matière de protection de la liberté individuelle ${ }^{22}$. Dès lors que la censure du législateur est désormais réservée aux erreurs manifestes ou atteintes manifestement

6. Ou constitutionnel, comme celui de l'article 16 de la Constitution de 1958.

7. Pour des éléments de droit comparé, voir, dans le présent numéro, notamment l’article de W. Mastor, «L'état d'exception aux États-Unis: le USA Patriot Act et autres violations "en règle" de la Constitution".

8. Loi n ${ }^{\circ}$ 2001-1062 du 15 novembre 2001 relative à la sécurité quotidienne, JORF, 16 novembre 2001, p. 18215.

9. Loi n ${ }^{\circ}$ 2003-239 du 18 mars 2003 pour la sécurité intérieure, JORF, 19 mars 2003, p. 4761.

10. Loi nº 2004-204 du 9 mars 2004 portant adaptation de la justice aux évolutions de la criminalité, JORF, 10 mars 2004, p. 4567.

11. Loi $\mathrm{n}^{\circ}$ 2006-64 du 23 janvier 2006 relative à la lutte contre le terrorisme et portant dispositions diverses relatives à la sécurité et aux contrôles frontaliers, JORF, 2006, p. 1129, commentaire F. Rolin, S. Slama, « Les libertés dans l'entonnoir de la législation antiterroriste », AJDA, 2006, p. 975-982.

12. Cette clause du rendez-vous est applicable à ses articles 3, 6 et 9.

13. D. Bigot, "L'impact des mesures anti-terroristes: sur l'équilibre entre liberté et sécurité et sur la cohésion sociale en France», cité par F. Rolin, S. Slama, «Les libertés dans l’entonnoir de la législation antiterroriste», p. 976.

14. P. Hassner, «L'état d'exception permanent », Le Monde, 24 juin 2003.

15. G. Agamben, L'État d'exception. Homo sacer, Paris, Seuil, 2003.

16. CC 76-75 DC, 12 janvier 1977, «Fouille des véhicules », RJC, I, p. 45, considérant 5.

17. Article 66 de la Constitution de 1958 : «Nul ne peut être arbitrairement détenu. L’autorité judiciaire, gardienne de la liberté individuelle, assure le respect de ce principe dans les conditions prévues par la loi».

18. Rappelons qu'elle est à l'origine de la loi du 15 novembre 2001.

19. Aux dires des médias et instituts de sondage.

20. CC 2003-467 DC, 13 mars 2003, Loi pour la sécurité intérieure, JORF, 19 mars 2003, p. 4789.

21. CC 2005-532 DC, 19 janvier 2006, Loi relative à la lutte contre le terrorisme et portant dispositions diverses relatives à la sécurité et aux contrôles frontaliers, http://www.conseil-constitutionnel.fr.

22. Pour plus de détails, voir G. Armand, «Que reste-t-il de la protection constitutionnelle de la liberté individuelle? », RFDC, $\mathrm{n}^{\circ}$ 65, 2006-1, p. 37-72. 
disproportionnées ou excessives qu'il porterait à cette liberté, il ne faut pas être étonné de voir le Haut Conseil «laisser passer le chameau ${ }^{23}$ et permettre l'extension inconsidérée des régimes de pouvoirs exceptionnels (I). Certes, l'intervention constitutionnellement obligatoire de l'autorité judiciaire est présentée comme permettant de compenser cette mise en cause des principes essentiels de la liberté individuelle ${ }^{24}$. Mais cette garantie judiciaire fait l'objet dans le même temps d'un tel affaiblissement que l'on peut légitimement se demander ce qui sépare encore les régimes exceptionnels en période normale de ceux adoptés en période exceptionnelle (II).

\section{L'extension des pouvoirs exceptionnels}

La notion de pouvoirs exceptionnels est familière au Conseil constitutionnel. Ainsi, dès la décision du 12 janvier 1977 Fouille des véhicules ${ }^{25}$, il a estimé que « la mise en vigueur d'un régime légal de pouvoirs exceptionnels» permettait de tenir en échec les principes essentiels de la liberté individuelle, avant de juger dans la décision du 25 janvier $1985^{26}$ que l'instauration de l'état d'urgence en Nouvelle-Calédonie était conforme à la Constitution alors même que, contrairement à l'état de siège, ce régime n'est pas prévu par le texte constitutionnel.

Mais le juge ne s'est pas arrêté en si bon chemin puisqu’il a considéré que, même en dehors des périodes exceptionnelles, des circonstances particulières tenant à la gravité et à la complexité de certaines infractions justifiaient l'établissement de procédures dérogatoires. Le problème est que ces procédures bis sont gloutonnes ${ }^{27}$ et ne cessent de dévorer le droit commun des libertés. En premier lieu, parce que le juge constitutionnel ne parvient pas (mais le veut-il ?) à limiter leur champ d'application à certaines infractions. Il s'agit donc de pouvoirs extensibles (A). En second lieu, parce que les pouvoirs conférés aux autorités de police sont si importants qu'on a le sentiment, assez désagréable, que rien n'est par principe interdit par la Constitution. Puisque tout est donc permis pourvu que les circonstances le justifient, nous sommes donc en présence aussi de pouvoirs étendus (B).

\section{A. Des pouvoirs extensibles}

C'est dans la politique de l'amalgame développée par le législateur avec l'aval du Conseil constitutionnel (1) qu'il faut rechercher la raison de la contamination du droit commun par le droit dérogatoire (2).

\section{La politique de l'amalgame}

Le traitement réservé aux infractions terroristes, en France comme outre-Manche et outre-Atlantique, est révélateur de cette politique de l'amalgame. Ainsi, il transparaît de la législation française adoptée depuis le début des années 2000 que le Parlement est animé par la volonté de ne pas lutter isolément contre le terrorisme, mais aussi contre les infractions qui l'alimentent. Mais devant l'épineuse difficulté à définir ces infractions, il est conduit par précaution à considérer que tout ou presque peut venir nourrir le terrorisme et justifier l'établissement de procédures exceptionnelles de contrainte sur les biens ou les personnes.

Subissant le choc de l'effondrement des tours jumelles, la loi relative à la sécurité quotidienne du 15 novembre 2001 était pourtant restée prudente à cet égard en limitant le champ d'application du droit dérogatoire aux infractions en matière d'armes et d'explosifs, terrain d'action privilégié de ceux qui entendent provoquer la terreur, ainsi qu'au trafic de stupéfiants ${ }^{28}$, dont on sait qu'il constitue une manne financière appréciée des terroristes qui se délectent de ses profits. Mais dès la loi pour la sécurité intérieure du 18 mars 2003, on allait assister à un «transfert de légitimité de l'anti-terrorisme » ${ }^{29}$ vers d'autres secteurs, en l'occurrence celui du vol et du recel, ajoutés comme justification à l'exercice des pouvoirs exceptionnels de visites des véhicules ${ }^{30}$. Amplifiant le mouvement, la loi Perben II du 9 mars 2004 devait par la suite considérer que le droit (dérogatoire?) applicable en matière de perquisitions domiciliaires pouvait être étendu à une liste de 17 infractions ${ }^{31}$, englobées sous le terme générique de «criminalité et délinquance organisées », et dont certaines, comme l'aide à l'entrée et au séjour irréguliers d'un étranger, n'entretiennent qu'un rapport lointain avec la menace terroriste, sauf à considérer que l'étranger, qui vient d'ailleurs et ne fait pas partie de notre groupe national, inspire par nature la terreur.

Mais n'y a-t-il pas là une source d'inspiration à la politique de l'amalgame pratiquée par le législateur? La question mérite en tout cas d'être posée au vu du mélange des genres pratiqué par la législation récente et qui se décline sur une palette aux multiples facettes. On est ainsi en présence, tantôt de lois relatives au terrorisme mais contenant des dispositions qui ne lui sont pas spécifiques ${ }^{32}$,

23. J. Rivero, «Liberté individuelle et fouille des véhicules, note sous la décision du 12 janvier 1977 », in Le Conseil constitutionnel et les libertés, 2 e éd., Paris - Aix-en-Provence, Économica - Presses universitaires d'Aix-Marseille (Droit public positif), 1987, p. 74.

24. Compensation critiquable car elle revient à faire du contrôle judiciaire l'unique garantie de la liberté individuelle.

25. Précitée, considérant 4.

26. CC 85-187 DC, 25 janvier 1985, «État d'urgence en Nouvelle-Calédonie », RJC, I, p. 223.

27. Pour reprendre l'expression de C. Lazerges, «Le Conseil constitutionnel acteur de la politique criminelle», Rev. Sc. Crim., 2004-3, p. 736.

28. Voir les articles 23 et 24 de la loi.

29. F. Rolin, S. Slama, «Les libertés dans l'entonnoir de la législation antiterroriste », p. 976.

30. Article $78-2-2$ CPP.

31. Voir l'article 706-73 du Code de procédure pénale (CPP).

32. Le titre de la loi du 23 janvier 2006 «relative à la lutte contre le terrorisme et portant dispositions diverses relatives à la sécurité et aux contrôles frontaliers ", ainsi que ses articles 3,6 et 8 sont à cet égard révélateurs. 
étant précisé que dans le droit commun amalgamé, la lutte contre l'immigration clandestine occupe une place de choix ${ }^{33}$; tantôt de lois étrangères aux actes terroristes mais s'inspirant de ses procédures dérogatoires ${ }^{34}$. Est alors clairement affichée l'intention de voir l'État de droit contaminé par les pouvoirs exceptionnels.

\section{La contamination du droit commun par le droit dérogatoire}

C'est aussi bien en amont qu'en aval que le droit dérogatoire vient sournoisement s'instiller dans le droit commun.

En amont, le rayonnement - irradiation convient mieux s'agissant d'une législation aussi funeste - provient de la difficulté voire de l'impossibilité à définir les infractions qui appellent des recherches particulières compte tenu de leur gravité ou de la complexité à en poursuivre les auteurs. On se trouve alors en présence d'infractions fourre-tout dont l'élasticité favorise l'extension inconsidérée des pouvoirs exceptionnels. La criminalité et la délinquance organisées constituent une illustration topique. En effet, reprenant la méthode initiée pour le terrorisme ${ }^{35}$, le législateur n'a pas créé une infraction nouvelle mais une catégorie nouvelle susceptible de couvrir une multitude d'infractions à partir du moment où elles répondent à l'unique dénominateur commun prévu par la loi, à savoir la commission en bande organisée. Rien ou presque ne peut dès lors empêcher le législateur d'étendre la liste des infractions relevant de ce régime dérogatoire, ce dont il ne s'est d'ailleurs pas privé en y ajoutant le délit d'escroquerie commis en bande organisée par la loi du 5 mars 2007 relative à la prévention de la délinquance ${ }^{36}$.

Déjà si peu encadrés, il se trouve que les régimes exorbitants du droit commun peuvent de surcroît être développés en aval par leur application à des infractions autres que celles pour lesquelles leur institution a en principe été justifiée. Ce développement est possible à partir du moment où le législateur est venu légaliser de véritables détournements de procédure, par une règle qui est devenue d'application constante et sur laquelle le Conseil constitutionnel a curieusement fermé les yeux. En effet, la loi du 10 août 1993 a prévu que le fait que les opérations coup de poing, c'est-à-dire les contrôles d'identité diligentés sur réquisition du procureur de la République, révèlent des infractions autres que celles visées par ce magistrat, ne constitue pas une cause de nullité des procédures incidentes ${ }^{37}$. La règle a ensuite été transposée aux contrôles
Schengen ${ }^{38}$, puis étendue aux visites de véhicules par la loi du 18 mars $2003^{39}$, ainsi qu'en matière de perquisitions domiciliaires nocturnes par la loi du 9 mars $2004^{40}$.

Pour s'en tenir à ce dernier domaine, le danger de la règle est immédiatement perceptible puisque s'il est officiellement interdit, à peine de nullité, par la loi Perben II de procéder à des perquisitions de nuit pour la recherche et la constatation d'autres infractions que celles entrant dans le champ de la criminalité et de la délinquance organisées $^{41}$, dans le même temps la découverte, par hasard, d'autres infractions ne constitue pas une cause de nullité des procédures incidentes. Force est alors de reconnaître que les pouvoirs exceptionnels d'intrusion dans la vie privée prévus par cette législation sont mis au service des pouvoirs généraux détenus par les autorités de police, qui n'ont pu découvrir les infractions de droit commun et en poursuivre ensuite les auteurs qu'en détournant la procédure prévue par le droit dérogatoire de sa finalité première et en faisant une application, que l'on peut considérer comme abusive, des pouvoirs étendus qu'il contient.

\section{B. Des pouvoirs étendus}

Les pouvoirs exceptionnels se présentent tels des matriochka: de dimension exponentielle par rapport au droit commun (1), ils recèlent en leur sein une multitude de facettes qui les rendent géométriquement variables (2).

\section{Des pouvoirs à dimension exponentielle}

On retrouve ici une question classique en matière de droits et libertés qui est celle des limites aux limites et consistant en l'espèce à se demander s'il existe, en période normale, un seuil de constitutionnalité infranchissable par le législateur, seuil qui, dans l'affirmative, permettrait encore de distinguer les pouvoirs rendus permanents de ceux applicables uniquement en période exceptionnelle. Qu'il soit permis d'en douter au vu de la faiblesse des barrières territoriales et temporelles qui sont dressées par la Constitution et son juge à l'extension des pouvoirs exceptionnels en matière de liberté individuelle.

Tandis que le Conseil d'État précise qu'il est de la nature même d'un régime légal de pouvoirs exceptionnels d'être limité dans l'espace, on peut être étonné de voir le Conseil constitutionnel ne pas s'opposer à la légalisation de zones dangereuses ${ }^{42}$ extrêmement vastes et affranchies du respect des règles de droit commun protégeant les libertés. C’est la caractéristique première des

33. Voir l'article 7 de la loi de 2006.

34. Lois du 18 mars 2003 et du 9 mars 2004.

35. Voir N. Haupais, Les Réponses juridiques au terrorisme en France, Mémoire de DEA de droit public, Université de Caen, 1996, 148 p. (dactyl.).

36. Loi $\mathrm{n}^{\circ}$ 2007-297 du 5 mars 2007 relative à la prévention de la délinquance, JORF, 7 mars 2007, p. 4297.

37. Article 78-2 alinéa 2 CPP.

38. Réalisés dans les gares, ports et aéroports ouverts au trafic international ainsi que dans une bande de 20 kilomètres en deçà de la frontière de la France avec les États parties à la Convention de Schengen (article 78-2 alinéa 4 CPP).

39. Article 78-2-2 CPP.

40. Article 706-93 CPP.

41. Ibid. Le Conseil constitutionnel a mis l'accent sur cette garantie en matière de lutte contre le terrorisme: CC, 96-377 DC, 16 juillet 1996, « Perquisitions de nuit ", RJC, I, p. 671, considérant 17 .

42. Refusée par la Cour de cassation en matière de contrôles d'identité : voir Crim., 4 octobre 1984, Kandé, Bull. crim., nº 287, p. 767. 
contrôles d'identité Schengen, effectués dans les zones du même nom, et dont le développement tentaculaire fait peu à peu reculer le régime général applicable en la matière ${ }^{43}$. On se rappelle qu'à l'origine ${ }^{44}$ ces contrôles n'étaient autorisés que dans les gares, ports et aéroports ouverts au trafic international, ainsi que dans une bande de 20 kilomètres en deçà de la frontière de la France avec les États parties à la Convention de Schengen. Mais la loi du 23 janvier 2006 relative à la lutte contre le terrorisme a permis, dans l'une de ces dispositions qui n'est pas spécifique à cette lutte ${ }^{45}$, que lorsque le contrôle a lieu à bord d'un train effectuant une liaison internationale, il puisse être opéré jusqu'au premier arrêt se situant au-delà des 20 kilomètres de la frontière... voire en cet arrêt et un arrêt situé dans la limite des 50 kilomètres suivants! L'absence de censure par le juge constitutionnel de cette extension ${ }^{46}$ n'est pas si paradoxale qu'il pourrait paraître de prime abord. Certes, dans la décision du 5 août $1993^{47}$, le Conseil avait déclaré contraire à la liberté individuelle une possibilité d'extension desdites zones à 40 kilomètres. Mais outre que la censure s'expliquait par la compétence attribuée en l'espèce au pouvoir réglementaire, il avait été fait réserve « d'impératifs constants et particuliers de la sécurité publique», condition que le juge a certainement estimé remplie pour fermer les yeux sur la législation nouvelle.

Des pouvoirs exceptionnels exercés dans des territoires étendus... mais aussi durant des périodes de plus en plus longues, comme l'atteste le régime des perquisitions nocturnes du domicile. Depuis la loi du 9 mars 2004, ces perquisitions peuvent en effet être mises en œuvre pour la répression de l'ensemble des infractions qui relèvent de la criminalité et de la délinquance organisées et durant toutes les phases de la procédure pénale : non seulement dans le court moment ${ }^{48}$ de la flagrance ${ }^{49}$, comme l'avait déjà admis le Conseil constitutionnel en matière de terrorisme ${ }^{50}$; mais aussi - c'est une nouveauté - dans le cadre de l'enquête préliminaire, à la condition cependant que les locaux d'habitation soient exclus de ce type de perquisitions ${ }^{51}$; dans la phase d'instruction enfin, y compris cette fois-ci dans les locaux d'habitation ${ }^{52}$. Même si cette dernière possibilité n'est ouverte que dans des hypothèses de flagrance ou de quasi-flagrance ${ }^{53}$, elle crée indiscutablement une brèche dans les principes essentiels protecteurs de la liberté individuelle, qui se trouvent modulés en fonction des circonstances d'exercice des pouvoirs exceptionnels.

\section{Des pouvoirs à géométrie variable}

Parmi les infractions justifiant la mise en place de procédures de répression exceptionnelles, le législateur est soucieux d'en isoler certaines auxquelles il réserve un traitement privilégié.

C'est ainsi qu'au sein de la criminalité et de la délinquance organisées, le droit dérogatoire se trouve encore durci pour les auteurs d'infractions de trafic de stupéfiants et de terrorisme, lesquels ne peuvent par exemple bénéficier du droit de s'entretenir avec un avocat qu'à l'issue de la $72^{\mathrm{e}}$ heure de garde à vue $\mathrm{e}^{54}$. Mais le terroriste fait encore plus peur au législateur que le dealer. Preuve en est le régime appliqué à celui-là en matière de durée de la garde à vue. Alors que dans le droit commun, cette durée est limitée à 48 heures, portée à 96 heures en matière de criminalité et de délinquance organisées, la loi du 23 janvier 2006 a prévu une possibilité de prolongation supplémentaire de 48 heures de la garde à vue des présumés terroristes (soit 6 jours au total) lorsqu’il « existe un risque sérieux de l'imminence d'une action terroriste en France ou à l'étranger ou que les nécessités de la coopération internationale le requièrent impérativement ${ }^{55}$. Alors que les conditions posées par cette disposition sont générales et imprécises ${ }^{56}$, elle n'a pas été censurée par le Conseil constitutionnel dans sa décision du 19 janvier 2006 : peut-être, mais on sait que ce n'est pas une excuse, parce qu'il n'en a pas été saisi par une opposition qui, au moins inconsciemment, adhère à la logique sécuritaire ${ }^{57}$; probablement

43. Ce que confirme la Cour de cassation en considérant que dans ces zones la condition liée à la présence de circonstances particulières établissant la nécessité du contrôle d'identité n'est pas applicable: Cass., $2^{\mathrm{e}}$ civ., 23 mai 2001, D, 2002, $\mathrm{n}^{\mathrm{o}}$ 12, J, p. 992, note E. Cornut.

44. Loi du 10 août 1993 .

45. Article 3.

46. Qu'il aurait pu examiner d'office dans la décision du 19 janvier 2006.

47. CC 93-323 DC, 5 août 1993, "Contrôles d'identité », RJC, I, p. 535.

48. Quoique l'enquête de flagrance puisse durer 8 jours, voire se poursuivre jusqu'à 16 jours pour les crimes ou délits punis d'une peine égale ou supérieure à 5 ans (article 53 alinéa $3 \mathrm{CPP}$ ).

49. Article 706-89 CPP.

50. CC 96-377 DC, 16 juillet 1996, «Perquisitions de nuit », précitée.

51. Article 706-90 CPP.

52. Article 706-91 CPP.

53. Risque immédiat de disparition des preuves ou des indices matériels par exemple.

54. Article 706-88 CPP, au lieu de la $48^{\mathrm{e}}$ heure pour les autres infractions relevant de la criminalité et de la délinquance organisées.

55. Article 706-88 CPP issu de l'article 17 de la loi.

56. À l'heure de l'hyperterrorisme, y a-t-il encore un moment où n'existe pas « un risque sérieux de l'imminence d'une action terroriste en France ou à l'étranger » ? Comment l'autorité judiciaire peut-elle porter une appréciation sur «les nécessités de la coopération internationale»?

57. Le texte de saisine du 23 décembre 2005 est sur ce point éloquent puisque, «afin d'éviter toute mauvaise compréhension de la saisine, ses auteurs entendent rappeler leur attachement républicain à la poursuite des objectifs de sauvegarde de l'ordre public et de recherche des auteurs d'infractions, essentiels à toute société démocratique. Qu'il en va particulièrement ainsi en matière de lutte contre les actes de terrorisme, qu'il s'agisse de la prévention ou de la répression de ces crimes odieux». Comment ne pas lier à ce dédouanement devant l'opinion publique l'absence de moyens développés par les requérants à l'égard des dispositions les plus liberticides de la loi, ainsi que le manque de rigueur juridique édifiant de leur argumentation? Ex : "l'autorité judiciaire gardienne des libertés individuelles aux termes de l'article 66 de la Constitution », «la protection de la liberté individuelle [...] repose aussi sur l'article 16 de la Déclaration de 1789 ", etc. 
aussi parce que le juge a déjà admis que la rétention administrative des étrangers pouvait être portée à 32 jours sans violer la Constitution ${ }^{58}$. Comment dès lors filtrer le moustique après avoir laissé passer le chameau et, puisque la boîte de Pandore a été ouverte dans le laboratoire constitutionnel que constitue le contentieux des étrangers et du terrorisme, il n'y a aucune raison à ce qu'elle se referme dans d'autres domaines ${ }^{59}$.

Puisqu'aucune limite matérielle (nature des infractions), territoriale ou temporelle, n'est apparemment infranchissable par le législateur instituant des pouvoirs exceptionnels, on peut légitimement se demander ce qu'il reste des principes essentiels de la liberté individuelle en période normale. Si ce n'est que la sauvegarde de ces principes est placée entre les mains de l'autorité judiciaire, comme l'illustre avec acuité la législation sur les opérations coup de poing ${ }^{60}$. Mais est-il légitime d'accorder une telle confiance à une autorité dont le domaine et les conditions d'intervention apparaissent tant affaiblis?

\section{L'affaiblissement de la garantie judiciaire}

L'indépendance constitutionnellement reconnue à l'autorité judiciaire et l'effectivité du contrôle qu'elle met en ouvre constituent les fondements de l'article 66 de la Constitution et conduisent aujourd'hui à considérer que la garantie judiciaire est devenue "l'alpha et l'oméga " ${ }^{61}$ de la liberté individuelle, capable de constituer à elle seule un rempart contre l'usage illimité des pouvoirs exceptionnels. Mais pour qu'il s'agisse d'une garantie efficace, encore faut-il qu'elle trouve à s'appliquer et que l'intervention de l'autorité judiciaire, lorsqu'elle est obligatoire, soit de qualité. Or c'est ce dont on peut précisément douter à l'heure où l'on assiste simultanément à une réduction du champ d'application (A) et à un appauvrissement (B) de la garantie judiciaire.

\section{A. Une réduction de la garantie judiciaire}

Il ne faudrait pas croire que l'intégralité des mesures exorbitantes du droit commun adoptées au nom de la lutte contre les infractions qui constituent une menace particulière pour l'ordre public est placée sous contrôle judiciaire.
Ce serait oublier que l'application de la garantie judiciaire est conditionnée par une atteinte à la liberté individuelle au sens de l'article 66 de la Constitution, atteinte que le juge constitutionnel s'évertue à circonscrire au fil du temps. Réduite à une peau de chagrin, la liberté individuelle peut alors laisser place au développement d'une législation d'exception soustraite au contrôle judiciaire (1) et uniquement placée sous contrôle administratif (2).

\section{Des pouvoirs exceptionnels sans contrôle judiciaire}

Comme nous avons déjà eu l'occasion de l'exposer ${ }^{62}$, l'histoire de la notion de liberté individuelle est celle d'un roman aux multiples chapitres et rebondissements. Consacrée à l'origine en tant que principe fondamental reconnu par les lois de la République ${ }^{63}$ et limitée à ses composantes historiques (sûreté et inviolabilité du domicile), la liberté individuelle a ensuite été surdimensionnée au point d'être assimilée au principe général de liberté (personnelle) consacré par la Déclaration de 1789 et d'inclure la plupart des libertés individuelles. Suivant la logique du point de rencontre $^{64}$, le Conseil constitutionnel a depuis mené une politique de resserrement de la notion en décidant de rattacher la liberté d'aller et venir et le droit au respect de la vie privée aux articles 2 et / ou $4{ }^{65}$ de la Déclaration, étant précisé qu'une mise en cause de ces droits pourrait les faire rencontrer le champ de l'article 66 de la Constitution.

Cette logique semble abandonnée par la jurisprudence récente, qui opère une restriction plus poussée du domaine de la liberté individuelle et partant de la garantie judiciaire qui lui est consubstantielle. Il semble ressortir notamment de la jurisprudence sur la loi relative à la lutte contre le terrorisme du 19 janvier 2006 déjà citée que le principe de liberté individuelle énoncé à l'article 66 alinéa 2 de la Constitution est réduit à la seule règle de l'alinéa $1^{\text {er }}$ portant prohibition des détentions arbitraires. Cette identification résulte de la décision elle-même ${ }^{66}$ et plus visiblement encore du commentaire du secrétaire général du Conseil qui, en tant qu'interprète authentique et officiel, peut affirmer que «la liberté individuelle (au sens de l'article 66 de la Constitution) s'entend de la liberté de ne pas être arbitrairement détenu (habeas corpus) ${ }^{67}$.

58. CC 2003-484 DC, 20 novembre 2003, Loi relative à la maîtrise de l'immigration, au séjour des étrangers en France et à la nationalité, JORF, 27 novembre 2003 , p. 20154 .

59. Comme cela s'est déjà produit par le passé, il faut s'attendre à ce que la garde à vue de 6 jours prévue par l'article 17 de la loi de 2006 , qui n'est pas concerné par la clause du rendez-vous, soit étendue à l'ensemble de la criminalité et de la délinquance organisées... en attendant la suite.

60. En vertu de l'article 78-2 alinéa 2 CPP, c'est en effet le procureur de la République qui précise les infractions visées par ces opérations, ainsi que la période de temps et le lieu de leur exercice.

61. E. Picard, «Les contrôles d’identité au regard des droits fondamentaux : des régimes inutilement hétéroclites », RFDA, 1994 , p. 969.

62. Voir G. Armand, L'Autorité judiciaire, gardienne de la liberté individuelle, dans la jurisprudence du Conseil constitutionnel, Thèse dactylographiée, Université de Caen, 28 octobre 2000.

63. CC 76-75 DC, 12 janvier 1977, «Fouilles des véhicules », précitée.

64. Droit constitutionnel, L. Favoreu (dir.), $7^{\mathrm{e}}$ éd., Paris, Dalloz (Droit public et science politique), 2004, p. 793.

65. Articles 2 et 4 pour la liberté d'aller et de venir, article 2 uniquement pour la vie privée, sans qu’on connaisse précisément les raisons de cette différence.

66. Voir en particulier le considérant 16

67. J.E. Schoettl, «Commentaire de la décision $\mathrm{n}^{\circ}$ 2005-532 du 19 janvier 2006, loi relative à la lutte contre le terrorisme et portant dispositions diverses relatives à la sécurité et aux contrôles frontaliers ", Cahiers $d u$ Conseil constitutionnel, $\mathrm{n}^{\circ}$ 20, http://www.conseil-constitutionnel.fr. 
Appliquée à l'espèce, elle conduit à considérer que certaines dispositions de la loi du 23 janvier 2006, telles les procédures de réquisition de données électroniques ${ }^{68}$ ou de recueil automatisé de données relatives aux véhicules ${ }^{69}$, sont affranchies du respect de la garantie judiciaire, alors même qu'elles sont adoptées dans un contexte exceptionnel de lutte contre le terrorisme.

\section{Des pouvoirs exceptionnels sous contrôle administratif}

Soustraites au contrôle judiciaire, les libertés n'en sont pas pour autant dépourvues de toute garantie constitutionnelle, qu'elle soit assurée par l'intervention de commissions administratives ou du juge administratif.

L'intervention de commissions administratives n'est pas sans soulever de difficultés dans la mesure où le Conseil constitutionnel ne parvient pas à leur appliquer un principe d'équivalence des garanties supposant qu'elles offrent une protection, sinon identique, du moins équivalente à celle procurée par l'autorité judiciaire. Il en va ainsi de leur indépendance, sujette à caution, mais aussi et surtout de l'effectivité du contrôle qu'elles mettent en œuvre. Ainsi, la Commission départementale compétente en matière de vidéosurveillance ${ }^{70}$ ou la Commission nationale de contrôle des interceptions de sécurité (CNCIS) intervenant en matière d'écoutes téléphoniques ne disposent jamais d'un pouvoir de contrainte leur permettant de s'opposer aux atteintes à la vie privée, leurs avis étant purement consultatif. De plus, l'intervention de ces commissions peut n'être qu'indirecte, comme l'atteste la loi du 23 janvier 2006, dont l'article 6 a prévu que la CNCIS désigne, sur proposition du ministre de l'Intérieur, une personnalité qualifiée compétente en matière de réquisitions de données conservées et traitées par les opérateurs de réseaux informatiques ${ }^{71}$. Enfin, ce contrôle peut être tout simplement écarté lorsque les circonstances le justifient, comme l'a décidé le législateur en 2006 en dispensant le représentant de l'État de recueillir l'avis de la Commission départementale compétente en matière de vidéosurveillance « lorsque l'urgence et l'exposition particulière à un risque d'actes de terrorisme le justifient ${ }^{72}$. Cette exclusion est paradoxale, alors que le contrôle judiciaire est au contraire renforcé lorsqu'il s'agit d'appliquer une législation pénale dérogatoire ${ }^{73}$ et que le Conseil d'État, en période d'application de l'état d'urgence, a insisté sur la garantie représentée par l'intervention d'une commission départementale de nature administrative en matière d'interdiction de séjour et d'assignation à résidence ${ }^{74}$.

Il ne reste plus alors qu'à croire en le juge administratif pour assurer le respect des libertés. Assurément, la confiance placée en celui-ci n'est pas imméritée, notamment depuis qu'il intervient efficacement grâce à la procédure du référé-liberté; et le Conseil constitutionnel ainsi que son secrétaire général ont raison lorsqu’ils vantent les mérites du dualisme juridictionnel apte à assurer la garantie des droits proclamée par l'article 16 de la Déclaration de $1789^{75}$. Mais ce n'est pas une raison pour oublier que contrairement à l'autorité judiciaire, le juge administratif n'intervient jamais a priori pour autoriser une atteinte à la liberté individuelle; et il n'est pas innocent que le Conseil d'État ait lui-même souligné l'importance de la garantie judiciaire en visant l'article 66 de la Constitution et en insistant sur la nécessité d'un contrôle de l'autorité judiciaire sur les mesures prises en application de l'état d'urgence $^{76}$.

\section{B. Un appauvrissement de la garantie judiciaire}

En l'état actuel du contentieux constitutionnel, il est rassurant de constater qu'en période normale les mesures les plus gravement attentatoires à la liberté individuelle adoptées sur le fondement de régimes exceptionnels demeurent placées sous le champ de la garantie judiciaire de l'article 66 de la Constitution. Mais le rassurement ne vaut pas pour autant réjouissance, compte tenu de l'appauvrissement dont font l'objet les notions d'autorité (1) et de contrôle (2) judiciaires.

\section{L'appauvrissement de l'autorité judiciaire}

Le principe d'unité du corps judiciaire ${ }^{77}$ ne doit pas masquer le fait que ce corps est représenté par deux catégories de magistrats aux statuts bien distincts même s'ils ont connu des rapprochements : d'un côté les magistrats du siège, dont l'indépendance est constitutionnellement reconnue, notamment par la règle d'inamovibilité consacrée à l'article 64 de la Constitution; de l'autre les magistrats du parquet, qui ne tirent pas bénéfice de cette règle et demeurent soumis au principe de subordination hiérarchique à l'égard de l'exécutif malgré la révision constitutionnelle du 27 juillet $1993^{78}$.

\footnotetext{
68. Article 6.

69. Article 8.

70. Article 10 de la loi no $95-73$ du 21 janvier 1995 d'orientation et de programmation relative à la sécurité.

71. Mais il est vrai que l'autorité ainsi désignée détient ici un véritable pouvoir de décision.

72. Article $1^{\text {er }}$ de la loi du 23 janvier 2006.

73. Intervention d'un magistrat du siège, et non du parquet, pour autoriser une perquisition nocturne ou la prolongation d'une garde à vue au-delà de 48 heures.

74. CE, Ord. Réf., 14 novembre 2005, Rolin, Rec., p. 499.

75. Voir la décision du 19 janvier 2006 et le commentaire aux Cahiers.

76. Voir les ordonnances précitées Rolin et Allouache.

77. Sur ce principe, voir T.-S. Renoux, «Le statut constitutionnel des juges du siège et du parquet en France », AIJC, XI, 1995 (Actes de la XI ${ }^{\mathrm{e}}$ Table ronde internationale des 15 et 16 septembre 1995), p. 227 sq.

78. Qui étend la compétence du Conseil supérieur de la magistrature à leur égard, mais selon des modalités d'intervention qui demeurent en retrait de celles applicables aux magistrats du siège.
} 
Le Conseil constitutionnel a pris acte de cette différence en opérant une répartition des compétences entre les magistrats du siège et du parquet, les derniers ne pouvant contrôler que certaines atteintes, les moins graves, à la liberté individuelle. Au fil du temps, on a cependant assisté à une extension des pouvoirs du parquet au détriment de ceux du siège, appauvrissant la qualité de la garantie attachée à l'intervention de l'autorité judiciaire. Ainsi, alors qu'ils n'intervenaient à l'origine que dans des domaines limités (surveillance des opérations de contrôle d'identité, prolongation de la garde à vue jusqu'à 48 heures), les procureurs ont par la suite été habilités à autoriser les opérations coup de poing ${ }^{79}$, les visites sommaires ${ }^{80}$ puis les véritables fouilles de véhicules ${ }^{81}$.

Il faut cependant reconnaître que les pouvoirs exceptionnels ne sont pas, pour le moment, affectés directement par l'évolution, des mesures telles que la prolongation de la garde à vue au-delà de 48 heures ou la perquisition domiciliaire nocturne applicables en matière de terrorisme et plus généralement de criminalité et de délinquance organisées ne pouvant être autorisées que par un magistrat du siège. Mais rien ne dit que la limite serait infranchissable si le Parlement se hasardait à prévoir la compétence des magistrats du parquet en la matière. Le juge constitutionnel pourrait l'admettre, à l'heure où l'on envisage une réforme du statut du parquet ${ }^{82}$.

\section{L'appauvrissement du contrôle judiciaire}

Le contrôle judiciaire est la dernière victime de l'affaiblissement de la contrainte de constitutionnalité. Par le passé, le Conseil constitutionnel s'était pourtant montré exigeant en la matière, employant les termes de " contrôle effectif ${ }^{83}$ et demandant à ce que l'autorité judiciaire autorise les opérations portant atteinte à la liberté individuelle, mais aussi en surveille concrètement le déroulement et puisse y mettre fin à tout moment. Mais, depuis la décision du 13 mars 2003 relative à la loi pour la sécurité intérieure, ce contrôle effectif et concret a fait place à un autre qui est beaucoup plus abstrait et relâché, consistant simplement en l'exercice d'une «surveillance » des mesures couvertes par l'article 66 de la Constitution ${ }^{84}$. Ce pouvoir général de surveillance confine finalement à celui qui est confié aux magistrats, du parquet essentiellement, par les articles 12 et suivants du Code de procédure pénale, et a conduit le Haut Conseil à faire preuve de bien peu de rigueur lorsqu'il a admis des dérogations au principe d'intervention préalable de l'autorité judiciaire en matière de fouilles des véhicules $^{85}$.
À partir de ces différents éléments, est-il possible d'affirmer que la liberté individuelle demeure porteuse de garanties constitutionnelles limitant les régimes exceptionnels en période normale et permettant de les distinguer des régimes légaux en période exceptionnelle? Peuton imaginer, pour reprendre l'exemple introductif, qu'une loi adoptée en dehors de l'état d'urgence ou de l'état de siège vienne légaliser l'exercice de perquisitions nocturnes du domicile sans les soumettre à l'autorisation préalable de l'autorité judiciaire? Aujourd'hui, assurément non, le Conseil constitutionnel continuant d'appliquer l'article 66 à ce type de mesure dérogatoire et mettant en valeur la qualité, du siège, attachée au magistrat qui l'autorise. Mais pour demain, la porte n'est peut-être pas fermée, et puisque rien n'apparaît constitutionnellement inadmissible, on peut dessiner quelques perspectives d'évolution risquant d'assombrir le ciel des libertés.

Ainsi, comme perspective la plus sombre, on peut imaginer que le juge constitutionnel ne considère plus l'inviolabilité du domicile comme une composante de la liberté individuelle au sens de l'article 66 de la Constitution, balayant ainsi corrélativement l'obligation d'intervention de l'autorité judiciaire. Depuis la décision du 19 janvier 2006 sur la loi relative à la lutte contre le terrorisme, l'évolution n'est pas irréaliste. En effet cette décision, on l'a vu, assimile la notion de liberté individuelle visée à l'alinéa 2 de l'article 66 à l'interdiction des détentions arbitraires prévue par son alinéa ${ }^{\text {er }}$, et si, dans son commentaire aux Cahiers, le secrétaire général réserve en plus l'hypothèse où « une législation républicaine constante ${ }^{86}$ impliquerait un contrôle judiciaire, rien ne dit qu'elle serait la source d'un PFRLR, donc à valeur constitutionnelle et s'imposant en tant que telle au législateur.

À moins que, de manière plus probable et moins obscure, les juges de Montpensier continuent d'appliquer l'article 66 de la Constitution à ce contentieux, mais en se contentant d'exiger que l'autorité judiciaire, du siège ou du parquet, exerce une surveillance générale des opérations de perquisitions de jour comme de nuit, surveillance n'impliquant pas nécessairement qu'elle les autorise de manière préalable. Mais on ne voit pas ce qui distinguerait encore ce régime exceptionnel en période normale de celui applicable en période exceptionnelle d'état d'urgence, dans la mesure où le juge administratif a, au visa de l'article 66, insisté sur le fait que les perquisitions autorisées par le ministre de l'Intérieur et le préfet sur le fondement de la loi de 1955 demeuraient des opérations de police judiciaire,

\footnotetext{
79. Loi du 5 août 1993.

80. Loi du 22 avril 1997.

81. Loi du 18 mars 2003.

82. Même si l'évolution est de faible portée, le projet de loi constitutionnelle de modernisation des institutions de la Ve République prévoit en effet que le Conseil supérieur de la magistrature pourrait désormais émettre un avis sur les nominations des procureurs généraux alors qu'il ne peut aujourd'hui le faire qu'à l'égard des procureurs et substituts.

83. CC 84-184 DC, 29 décembre 1984, RJC, I, p. 212, considérant 35.

84. CC2003-467 DC, 13 mars 2003, Loi pour la sécurité intérieure, précitée, considérants 8 et 10.

85. Pour plus de détails, voir G. Armand, «Que reste-t-il de la protection constitutionnelle de la liberté individuelle? », p. 70.

86. Commentaire précité.
} 
soumises en tant que telles au contrôle de l'autorité judiciaire $^{87}$.

Les pouvoirs exceptionnels légalisés en période normale rejoindraient alors ceux applicables dans les circonstances exceptionnelles, rendant ces derniers de facto inutiles et obsolètes. L'État de droit lui, ou ce qu'il en res- tera, se trouverait placé dans la situation de ce serpent qui s'est mordu la queue et qui, à vouloir défendre la liberté à n'importe quel prix, y a finalement renoncé par l'institution d'un état d'exception permanent.

Est-ce donc finalement pour un moment seulement que le voile peut être mis sur la liberté? 